\title{
Nonlinear Semi-Analytic Method for Spacecraft Navigation
}

\author{
Ryan S. Park *and Daniel J. Scheeres ${ }^{\dagger}$ \\ The University of Michigan, Ann Arbor, Michigan, 48109
}

\begin{abstract}
A nonlinear semi-analytic filtering method to sequentially estimate spacecraft states and their associated uncertainties is presented. We first discuss the state transition tensors that characterize the localized nonlinear behavior of the spacecraft trajectory and illustrate the importance of higher order effects on orbit uncertainty propagation. We then present the semi-analytic filtering method by implementing the state transition tensors to sequentially update the information with contributions from each measurement, which requires no integration once the tensors are computed. A Sun-Earth halo orbit about the $L_{1}$ point is considered as an example with realistic orbit uncertainties, and the results are compared with the extended Kalman filter and unscented Kalman filter.
\end{abstract}

\section{Introduction}

Orbit uncertainty propagation plays an important role in various space-related applications, such as orbit determination, ${ }^{1-3}$ parameter estimation, ${ }^{4-9}$ maneuver design,,${ }^{10-12}$ small-body collision/encounter analysis,${ }^{13}$ etc. In practice, it is usually assumed that the true state (in a statistical sense) is within a boundary where the linear assumption sufficiently approximates a trajectory dynamics and the covariance matrix is mapped using the Riccati equations. In some cases, however, the linear assumption fails to provide an accurate realization of the local trajectory motion, and in such cases, a different method, which accounts for the system nonlinearity, must be implemented.

The best known technique is a Monte-Carlo (MC) simulation, which approximates a nonlinear transformation by averaging a large set of nonlinearly propagated random samples. However, because of intensive numerical computation and challenging implementation, the Monte-Carlo technique is often not suitable for orbit uncertainty propagation in filtering applications. Recently, a simple method called the unscented transformation ${ }^{14-18}$ was proposed by Julier et al., which deterministically chooses the sample sigma points to capture the second order effect while keeping the computational cost at the same order as the linear method. As pointed out by Julier et al., the unscented transformation is based on the idea that, for a given system, it is easier to approximate the probability distribution than the nonlinear function. ${ }^{16,17}$ However, it is also true that if such approximations of the nonlinear function are feasible, they would provide a significant advantage over approximating the probability distribution.

For deep-space missions, one is usually given a reference (nominal) trajectory with precise ephemerides. The objective of trajectory navigation and spacecraft control is to follow the reference trajectory while minimizing some pre-defined optimality constraints, such as number of trajectory correction maneuvers, flight time, fuel, etc. The basic underlying concept of such a process is to stay within the linear region by taking sufficient number of measurements and linearly map the deviation and statistics via the state

\footnotetext{
* Graduate Student Research Assistant, PhD Candidate, Department of Aerospace Engineering, The University of Michigan, Ann Arbor, Michigan, AIAA Student Member, AAS Student Member, sanghp@umich.edu.

${ }^{\dagger}$ Associate Professor, Department of Aerospace Engineering, The University of Michigan, Ann Arbor, Michigan, Associate Fellow AIAA, Member AAS, scheeres@umich.edu.
} 
transition (fundamental) matrix with respect to the nominal trajectory. It is, however, not obvious to say if the true (in a statistical sense) trajectory is within the linear regime.

Conventionally, the extended Kalman filter (EKF) is used for spacecraft navigation and the batch leastsquares filter (LSF) is used for trajectory reconstructions. ${ }^{1-3,19,20}$ Except for a very few cases, these methods have provided sufficient accuracy for mission operations and science requirements. However, both the EKF and LSF are based on the linearized dynamics model, and when the nonlinearity is significant or when only a limited number of measurements are available, the filter solutions are often overestimated and the linear models become insufficient for precision analysis or robust navigation. For example, the orbit uncertainties are often believed to be overestimated for deep space missions and the covariances are arbitrarily increased (based on experience) to compensate for potential solution errors. Moreover, as technology advances, high accuracy measurements will be available, and hence, need for nonlinear filters will become more critical to level the filter performance with the measurement accuracy.

There exist various nonlinear filtering methods, such as the particle filter, ${ }^{21-23}$ unscented Kalman/particle filter (UKF/UPF) ${ }^{14-18,24}$ Gaussian sum filter,${ }^{25,26}$ divided difference filter, ${ }^{27}$ finite dimensional filters, ${ }^{28-33}$ and higher order filters. ${ }^{1,34}$ This paper discusses the significance of nonlinearity on spacecraft navigation and introduces a sub-optimal nonlinear semi-analytic method that sequentially estimates a spacecraft trajectory. The idea of the present paper is to extend the function of the state transition matrix (STM) by including the higher order effects, ${ }^{35,36}$ which we call the state transition tensors (STTs), ${ }^{10,11}$ and develop a higher order semi-analytic filter which requires no online integration.

The Sun-Earth halo orbit about the $L_{1}$ point based on the circular restricted three-body problem (CR3BP) is chosen as an example since the overall nonlinearity is small, but the trajectory is unstable and when the spacecraft is not sufficiently observed, the nonlinear effect can become significant. The proposed semi-analytic filter is compared with the extended Kalman filter and unscented Kalman filter with realistic orbit uncertainties.

\section{Higher Order Perturbation Analysis}

The motion of a spacecraft can be modeled with first order ordinary differential equations, given in tensor notation:

$$
\frac{d \mathbf{x}^{i}(t)}{d t}=\mathbf{g}^{i}[t ; \mathbf{x}(t)]
$$

where $\mathbf{g}[t ; \mathbf{x}(t)]$ represents the system dynamics vector with a dimension $n$ and $\mathbf{x}=\left\{\mathbf{x}^{i} \mid i=1, \cdots, n\right\}$ represents the spacecraft state vector with initial conditions $\mathbf{x}_{0}^{i}=\mathbf{x}^{i}\left(t_{0}\right)$. For the given $\mathbf{x}_{0}$, the solution flow, which maps the initial state to the current time $t_{k}$, is defined as

$$
\mathbf{x}^{i}\left(t_{k}\right)=\phi^{i}\left(t_{k} ; \mathbf{x}_{0}, t_{0}\right)
$$

The solution flow is governed by

$$
\begin{aligned}
\frac{d \phi}{d t} & =\mathbf{g}\left[t, \phi\left(t ; \mathbf{x}_{0}, t_{0}\right)\right] \\
\phi\left(t_{0} ; \mathbf{x}_{0}, t_{0}\right) & =\mathbf{x}\left(t_{0}\right)
\end{aligned}
$$

and the flow of a phase volume can be stated as

$$
\mathcal{B}\left(t_{k}\right)=\left\{\mathbf{x}\left(t_{k}\right) \mid \mathbf{x}\left(t_{k}\right)=\phi\left(t_{k} ; \mathbf{x}_{0}, t_{0}\right) \forall \mathbf{x}_{0} \in \mathcal{B}_{0}\right\}
$$

The inverse solution flow, which maps the current state to the epoch, can be obtained by applying a similar approach, and to distinguish between the direct and inverse flows, we define the inverse flow as

$$
\mathbf{x}_{0}^{i}=\psi^{i}\left(t_{k}, \mathbf{x}_{k} ; t_{0}\right)
$$

which is an integral of motion since $d \mathbf{x}_{0} / d t=0$. Combining the definitions of the forward and inverse solutions, an obvious, but important, identity exists

$$
\mathbf{x}_{0}=\psi\left[t_{k}, \phi\left(t_{k} ; \mathbf{x}_{0}, t_{0}\right) ; t_{0}\right]
$$


In this framework, define a local trajectory dynamics, $\delta \mathbf{x}$, by applying the Taylor series expansion about the reference (nominal) trajectory $\overline{\mathbf{x}}$, i.e., $\delta \mathbf{x}(t)=\mathbf{x}(t)-\overline{\mathbf{x}}(t)$. The $m$ th order solution and its time derivatives can be stated as

$$
\begin{aligned}
\delta \mathbf{x}^{i}\left(t_{k}\right) & =\sum_{p=1}^{m} \frac{1}{p !} \phi_{\left(t, t_{0}\right)}^{i, \gamma_{1} \cdots \gamma_{p}} \delta \mathbf{x}_{0}^{\gamma_{1}} \cdots \delta \mathbf{x}_{0}^{\gamma_{p}} \\
\delta \dot{\mathbf{x}}^{i}(t) & =\sum_{p=1}^{m} \frac{1}{p !} \mathbf{g}^{i, \gamma_{1} \cdots \gamma_{p}} \delta \mathbf{x}^{\gamma_{1}} \cdots \delta \mathbf{x}^{\gamma_{p}}
\end{aligned}
$$

where $\gamma_{j} \in\{1, \cdots, n\}$, subscripts $\gamma_{j}$ denote the $\gamma_{j}$ th component of the state vector, and

$$
\begin{aligned}
\phi_{\left(t, t_{0}\right)}^{i, \gamma_{1} \cdots \gamma_{p}} & =\frac{\partial^{p} \mathbf{x}^{i}}{\partial \mathbf{x}_{0}^{\gamma_{1}} \cdots \partial \mathbf{x}_{0}^{\gamma_{p}}} \\
\mathbf{g}^{i, \gamma_{1} \cdots \gamma_{p}} & =\left.\frac{\partial^{p} \mathbf{g}^{i}}{\partial \mathbf{x}^{\gamma_{1}} \cdots \partial \mathbf{x}^{\gamma_{p}}}\right|_{\mathbf{x}=\overline{\mathbf{x}}}
\end{aligned}
$$

We call the higher order partials of the solution flow (i.e., Eqn. (10) the state transition tensors, which map the initial deviations to the current time. Note that the first order case (i.e., $p=1$ ) reduces to the usual STM and Eqn. (11) is simply the Jacobian matrix.

The differential equations for the STTs can be obtained by substituting Eqn. (8) into Eqn. (9) and equating with the time derivative of Eqn. (8) and balancing terms of the same order in $\delta \mathbf{x}_{0} \cdot{ }^{10,11}$ The differential equations up to fourth order deviation are given in Eqns. (12-15).

$$
\begin{aligned}
\dot{\phi}^{i, a} & =\mathbf{g}^{i, \alpha} \phi^{\alpha, a} \\
\dot{\phi}^{i, a b} & =\mathbf{g}^{i, \alpha} \phi^{\alpha, a b}+\mathbf{g}^{i, \alpha \beta} \phi^{\alpha, a} \phi^{\beta, b} \\
\dot{\phi}^{i, a b c} & =\mathbf{g}^{i, \alpha} \phi^{\alpha, a b c}+\mathbf{g}^{i, \alpha \beta}\left(\phi^{\alpha, a} \phi^{\beta, b c}+\phi^{\alpha, a b} \phi^{\beta, c}+\phi^{\alpha, a c} \phi^{\beta, b}\right)+\mathbf{g}^{i, \alpha \beta \gamma} \phi^{\alpha, a} \phi^{\beta, b} \phi^{\gamma, c} \\
\dot{\phi}^{i, a b c d} & =\mathbf{g}^{i, \alpha} \phi^{\alpha, a b c d} \\
& +\mathbf{g}^{i, \alpha \beta}\left(\phi^{\alpha, a b c} \phi^{\beta, d}+\phi^{\alpha, a b d} \phi^{\beta, c}+\phi^{\alpha, a c d} \phi^{\beta, b}+\phi^{\alpha, a b} \phi^{\beta, c d}+\phi^{\alpha, a c} \phi^{\beta, b d}+\phi^{\alpha, a d} \phi^{\beta, b c}+\phi^{\alpha, a} \phi^{\beta, b c d}\right) \\
& +\mathbf{g}^{i, \alpha \beta \gamma}\left(\phi^{\alpha, a b} \phi^{\beta, c} \phi^{\gamma, d}+\phi^{\alpha, a c} \phi^{\beta, b} \phi^{\gamma, d}+\phi^{\alpha, a d} \phi^{\beta, b} \phi^{\gamma, c}+\phi^{\alpha, a} \phi^{\beta, b c} \phi^{\gamma, d}+\phi^{\alpha, a} \phi^{\beta, b d} \phi^{\gamma, c}\right. \\
& \left.+\phi^{\alpha, a} \phi^{\beta, b} \phi^{\gamma, c d}\right)+\mathbf{g}^{i, \alpha \beta \gamma \delta} \phi^{\alpha, a} \phi^{\beta, b} \phi^{\gamma, c} \phi^{\delta, d}
\end{aligned}
$$

The initial conditions of the STTs are $\phi_{\left(t_{0}, t_{0}\right)}^{i, a}=1$ if $i=a$ and zero otherwise. Once these STTs are computed, they serve a role identical to the STM except that higher order effects are now included, and thus, the solution is nonlinear. Therefore, a significance of the STTs is that the local nonlinear motion of a spacecraft trajectory can be mapped analytically and requires no integration.

The inverse series also exists and is defined as

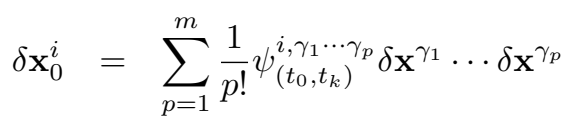

where $\gamma_{j} \in\{1, \cdots, n\}$ and we call $\psi_{\left(t_{0}, t_{k}\right)}^{i, \gamma_{1} \cdots \gamma_{p}}$ the inverse state transition tensors (ISTTs). The ISTTs can be computed by using the similar integration approach as in the STT computation; however, it is more convenient to compute via series reversion since the integration can take a long time for the large $m$. As functions of the STTs, the ISTTs mapping from $t_{k}$ to $t_{0}$ are

$$
\begin{aligned}
\psi_{k}^{i, a} & =\left[\boldsymbol{\Phi}^{-1}\left(t, t_{0}\right)\right]^{i, a} \\
\psi_{k}^{i, a b} & =-\psi_{k}^{i, \alpha} \phi_{k}^{\alpha, j_{1} j_{2}} \psi_{k}^{j_{1}, a} \psi_{k}^{j_{2}, b} \\
\psi_{k}^{i, a b c} & =-\left[\psi_{k}^{i, \alpha} \phi_{k}^{\alpha, j_{1} j_{2} j_{3}}+\psi_{k}^{i, \alpha \beta}\left(\phi_{k}^{\alpha, j_{1}} \phi_{k}^{\beta, j_{2} j_{3}}+\phi_{k}^{\alpha, j_{1} j_{2}} \phi_{k}^{\beta, j_{3}}+\phi_{k}^{\alpha, j_{1} j_{3}} \phi_{k}^{\beta, j_{2}}\right)\right] \psi_{k}^{j_{1}, a} \psi_{k}^{j_{2}, b} \psi_{k}^{j_{3}, c} \\
\psi_{k}^{i, a b c d} & =-\left[\psi_{k}^{i, \alpha} \phi_{k}^{\alpha, j_{1} j_{2} j_{3} j_{4}}+\psi_{k}^{i, \alpha \beta}\left(\phi_{k}^{\alpha, j_{1} j_{2} j_{3}} \phi_{k}^{\beta, j_{4}}+\phi_{k}^{\alpha, j_{1} j_{2} j_{4}} \phi_{k}^{\beta, j_{3}}+\phi_{k}^{\alpha, j_{1} j_{3} j_{4}} \phi_{k}^{\beta, j_{2}}+\phi_{k}^{\alpha, j_{1} j_{2}} \phi_{k}^{\beta, j_{3} j_{4}}\right.\right. \\
& \left.+\phi_{k}^{\alpha, j_{1} j_{3}} \phi_{k}^{\beta, j_{2} j_{4}}+\phi_{k}^{\alpha, j_{1} j_{4}} \phi_{k}^{\beta, j_{2} j_{3}}+\phi_{k}^{\alpha, j_{1}} \phi_{k}^{\beta, j_{2} j_{3} j_{4}}\right)+\psi_{k}^{i, \alpha \beta \gamma}\left(\phi_{k}^{\alpha, j_{1} j_{2}} \phi_{k}^{\beta, j_{3}} \phi_{k}^{\gamma, j_{4}}+\phi_{k}^{\alpha, j_{1} j_{3}} \phi_{k}^{\beta, j_{2}} \phi_{k}^{\gamma, j_{4}}\right. \\
& \left.\left.+\phi_{k}^{\alpha, j_{1} j_{4}} \phi_{k}^{\beta, j_{2}} \phi_{k}^{\gamma, j_{3}}+\phi_{k}^{\alpha, j_{1}} \phi_{k}^{\beta, j_{2} j_{3}} \phi_{k}^{\gamma, j_{4}}+\phi_{k}^{\alpha, j_{1}} \phi_{k}^{\beta, j_{2} j_{4}} \phi_{k}^{\gamma, j_{3}}+\phi_{k}^{\alpha, j_{1}} \phi_{k}^{\beta, j_{2}} \phi_{k}^{\gamma, j_{3} j_{4}}\right)\right] \psi_{k}^{j_{1}, a} \psi_{k}^{j_{2}, b} \psi_{k}^{j_{3}, c} \psi_{k}^{j_{4}, d}
\end{aligned}
$$


where $\psi_{k}=\psi_{\left(t_{0}, t_{k}\right)}$ and $\phi_{k}=\phi_{\left(t_{k}, t_{0}\right)}$ are used for the concise notations, and $\boldsymbol{\Phi}$ represents the usual STM. Note that Eqns. 17-20 are analytic in the STTs and require no integration. When computing the inverse of the STM in Eqn. (17), we can take the advantage of the symplectic structure of a Hamiltonian system, i.e.,

$$
\begin{aligned}
\boldsymbol{\Phi}^{-1} & =-\mathbf{T}^{-1} \mathbf{J} \mathbf{T}^{-T} \boldsymbol{\Phi}^{T} \mathbf{T}^{T} \mathbf{J} \mathbf{T} \\
\mathbf{T} & =\left[\begin{array}{cc}
\mathbf{I} & \mathbf{0} \\
\tilde{\omega} & \mathbf{I}
\end{array}\right] \\
\tilde{\omega} & =\left[\begin{array}{ccc}
0 & -1 & 0 \\
1 & 0 & 0 \\
0 & 0 & 0
\end{array}\right] \\
\mathbf{J} & =\left[\begin{array}{cc}
\mathbf{0} & \mathbf{I} \\
-\mathbf{I} & \mathbf{0}
\end{array}\right]
\end{aligned}
$$

where $\mathbf{I}$ and $\mathbf{J}$ are the identity and symplectic identity matrices, respectively, with proper dimensions.

By applying the forward and inverse state transition tensors, the STTs mapping from time $t_{r}$ to $t_{s}$, where $t_{r}, t_{s} \in\left[t_{0}, t_{f}\right]$ for some final time $t_{f}$ and $t_{r} \leq t_{s}$, can be represented as

$$
\begin{aligned}
\phi_{\left(t_{s}, t_{r}\right)}^{i, a} & =\left[\mathbf{\Phi}\left(t_{s}, t_{0}\right) \Phi^{-1}\left(t_{r}, t_{0}\right)\right]^{i, a}=\phi_{s}^{i, \alpha} \psi_{r}^{\alpha, a} \\
\phi_{\left(t_{s}, t_{r}\right)}^{i, b} & =\phi_{s}^{i, \alpha} \psi_{r}^{\alpha, a b}+\phi_{s}^{i, \alpha \beta} \psi_{r}^{\alpha, a} \psi_{r}^{\beta, b} \\
\phi_{\left(t_{s}, t_{r}\right)}^{i, a b c} & =\phi_{s}^{i, \alpha} \psi_{r}^{\alpha, a b c}+\phi_{s}^{i, \alpha \beta}\left(\psi_{r}^{\alpha, a} \psi_{r}^{\beta, b c}+\psi_{r}^{\alpha, a b} \psi_{r}^{\beta, c}+\psi_{r}^{\alpha, a c} \psi_{r}^{\beta, b}\right)+\phi_{s}^{i, \alpha \beta \gamma} \psi_{r}^{\alpha, a} \psi_{r}^{\beta, b} \psi_{r}^{\gamma, c} \\
\phi_{\left(t_{s}, t_{r}\right)}^{i, b c d} & =\phi_{s}^{i, \alpha} \psi_{r}^{\alpha, a b c d}+\phi_{s}^{i, \alpha \beta}\left(\psi_{r}^{\alpha, a b c} \psi_{r}^{\beta, d}+\psi_{r}^{\alpha, a b d} \psi_{r}^{\beta, c}+\psi_{r}^{\alpha, a c d} \psi_{r}^{\beta, b}+\psi_{r}^{\alpha, a b} \psi_{r}^{\beta, c d}+\psi_{r}^{\alpha, a c} \psi_{r}^{\beta, b d}\right. \\
& \left.+\psi_{r}^{\alpha, a d} \psi_{r}^{\beta, b c}+\psi_{r}^{\alpha, a} \psi_{r}^{\beta, b c d}\right)+\phi_{s}^{i, \alpha \beta \gamma}\left(\psi_{r}^{\alpha, a b} \psi_{r}^{\beta, c} \psi_{r}^{\gamma, d}+\psi_{r}^{\alpha, a c} \psi_{r}^{\beta, b} \psi_{r}^{\gamma, d}+\psi_{r}^{\alpha, a d} \psi_{r}^{\beta, b} \psi_{r}^{\gamma, c}\right. \\
& \left.+\psi_{r}^{\alpha, a} \psi_{r}^{\beta, b c} \psi_{r}^{\gamma, d}+\psi_{r}^{\alpha, a} \psi_{r}^{\beta, b d} \psi_{r}^{\gamma, c}+\psi_{r}^{\alpha, a} \psi_{r}^{\beta, b} \psi_{r}^{\gamma, c d}\right)+\phi_{s}^{i, \alpha \beta \gamma \delta} \psi_{r}^{\alpha, a} \psi_{r}^{\beta, b} \psi_{r}^{\gamma, c} \psi_{r}^{\delta, d}
\end{aligned}
$$

where $\psi_{r}=\psi_{\left(t_{0}, t_{r}\right)}$ are $\phi_{s}=\phi_{\left(t_{s}, t_{0}\right)}$ and the ISTTs are computed by applying the Eqns. (17-20). In other words, once the STTs are computed for the entire reference trajectory, the map from an arbitrary point in space to some future time becomes a simple algebraic manipulation. Note that $\phi_{\left(t_{s}, t_{r}\right)}^{i, \gamma_{p} \cdots \gamma_{p}}$ can also be computed by integrating the differential equations given in Eqns. (12-15) for each time interval $\left(t_{s}, t_{r}\right)$.

One concern is a numerical consistency when Eqns. (12-15) are integrated over a long duration of time. We note that the reference trajectory can be segmented arbitrarily to meet the desired numerical accuracy. Another question that may arise is the computational difficulty (or the integration time) as we consider the higher order solutions. Specifically, assuming a system with $n=6$, the $m$ th order analysis requires integration of $\sum_{q=1}^{m+1} 6^{q}$ equations. For example, when $m=3$, one must integrate 1554 equations simultaneously. However, the higher order solutions can be computed off-line, and especially when orbit is periodic (e.g., halo orbit), these only need to be computed once. Lastly, the computation of the partials of the dynamics may be of a concern. We note that there are symbolic manipulators available which provide optimal differentiations, and also note that many of these partials vanish to zero for systems of spacecraft navigation interest.

\section{Higher Order Extended Kalman Filter}

Suppose we are given the continuous trajectory model defined in Eqn. (3). Since a spacecraft tracking model is usually discrete, without loss of generality, consider the following discrete system model:

$$
\begin{aligned}
& \mathbf{x}_{k+1}=\phi\left(t_{k+1} ; \mathbf{x}_{k}, t_{k}\right)+\mathbf{w}_{k} \\
& \mathbf{z}_{k+1}=\mathbf{h}\left(\mathbf{x}_{k+1}, t_{k+1}\right)+\mathbf{v}_{k+1},
\end{aligned}
$$

where $\mathbf{x}_{k}$ is the true spacecraft state, $\phi$ is the solution flow, $\mathbf{w}_{k}$ is the white process noise perturbing the spacecraft state, $\mathbf{z}_{k}$ is the actual measurement, $\mathbf{h}$ is the measurement function, and $\mathbf{v}_{k}$ is the white measurement noise characterizing the observation error. The process noise and measurement noise are assumed to be non-correlated, i.e., $E\left[\mathbf{v}_{i} \mathbf{w}_{j}^{T}\right]=0$, with the autocorrelations

$$
\begin{aligned}
E\left[\mathbf{w}_{i} \mathbf{w}_{j}^{T}\right] & =\mathbf{Q}_{i} \delta_{i j} \\
E\left[\mathbf{v}_{i} \mathbf{v}_{j}^{T}\right] & =\mathbf{R}_{i} \delta_{i j}
\end{aligned}
$$


for all discrete time indexes $i$ and $j$, where $\delta_{i j}$ represents the Dirac delta function. Here, $\mathbf{Q}$ and $\mathbf{R}$ are also known as the diffusion and measurement noise matrices, respectively.

\section{A. Review of Gaussian Distribution}

Consider the spacecraft state as a Gaussian with a mean $\mathbf{m}$ and covariance matrix $\mathbf{P}$, i.e., $\mathbf{x} \sim \mathcal{N}(\mathbf{m}, \mathbf{P})$. The Gaussian probability density function (pdf) for $\mathbf{x}$ is then defined as

$$
p(\mathbf{x})=\frac{1}{\sqrt{(2 \pi)^{n} \operatorname{det} \mathbf{P}}} \exp \left[-\frac{1}{2}(\mathbf{x}-\mathbf{m})^{T} \mathbf{P}^{-1}(\mathbf{x}-\mathbf{m})\right]
$$

where, by definition, the first two moments are

$$
\begin{aligned}
\mathbf{m} & =E[\mathbf{x}] \\
\mathbf{P} & =E\left[\mathbf{x x}^{T}\right]-\mathbf{m m}^{T}
\end{aligned}
$$

and for an arbitrary nonlinear function $\mathbf{g}(\mathbf{x})$, the law of total expectation gives

$$
E[\mathbf{g}(\mathbf{x})]=\int_{\infty} \mathbf{g}(\xi) p(\xi) d \xi
$$

Note that $E[\cdot]$ represents the expectation operator.

It is a unique property of the Gaussian distribution that moments of any order for $\mathbf{x}$ are functions of $\mathbf{m}$ and $\mathbf{P}$, which can be computed using a joint characteristic function. ${ }^{37,38}$ For the Gaussian random vector the joint characteristic function is defined as

$$
\chi(\mathbf{u})=E\left[e^{j \mathbf{u}^{T} \mathbf{x}}\right]=\exp \left(j \mathbf{u}^{T} \mathbf{m}-\frac{1}{2} \mathbf{u}^{T} \mathbf{P u}\right)
$$

where $j=\sqrt{-1}$ and the higher moments can be computed using

$$
E\left[\mathbf{x}^{k_{1}} \mathbf{x}^{k_{2}} \cdots \mathbf{x}^{k_{m}}\right]=\left.j^{-m} \frac{\partial^{m} \chi(\mathbf{u})}{\partial \mathbf{u}^{k_{1}} \partial \mathbf{u}^{k_{2}} \cdots \partial \mathbf{u}^{k_{m}}}\right|_{\mathbf{u}=\mathbf{0}}
$$

\section{B. Extended Kalman Filter}

In the extended Kalman filter algorithm, the nominal trajectory is computed according to Eqn. (29) and the covariance matrix is linearly mapped assuming the Gaussian statistics: ${ }^{1-3}$

Prediction:

$$
\begin{aligned}
\mathbf{m}_{k+1}^{-} & =\phi\left(t_{k+1} ; \mathbf{m}_{k}^{+}, t_{k}\right) \\
\mathbf{P}_{k+1}^{-} & =\boldsymbol{\Phi}\left(t_{k+1}, t_{k}\right) \mathbf{P}_{k}^{+} \boldsymbol{\Phi}^{T}\left(t_{k+1}, t_{k}\right)+\mathbf{Q}_{k} \\
\mathbf{n}_{k+1}^{-} & =\mathbf{h}\left(\mathbf{m}_{k+1}^{-}, t_{k+1}\right)
\end{aligned}
$$

Update:

$$
\begin{aligned}
\mathbf{K}_{k+1} & =\mathbf{P}_{k+1}^{\mathbf{x z}}\left(\mathbf{P}_{k+1}^{\mathbf{z z}}\right)^{-1} \\
& =\mathbf{P}_{k+1}^{-} \mathbf{H}_{k+1}^{T}\left(\mathbf{H}_{k+1} \mathbf{P}_{k+1}^{-} \mathbf{H}_{k+1}^{T}+\mathbf{R}_{k+1}\right)^{-1} \\
\mathbf{m}_{k+1}^{+} & =\mathbf{m}_{k+1}^{-}+\mathbf{K}_{k+1}\left(\mathbf{z}_{k+1}^{*}-\mathbf{n}_{k+1}^{-}\right) \\
\mathbf{P}_{k+1}^{+} & =\mathbf{P}_{k+1}^{-}-\mathbf{K}_{k+1} \mathbf{P}_{k+1}^{\mathbf{z z}} \mathbf{K}_{k+1}^{T} \\
& =\mathbf{P}_{k+1}^{-}-\mathbf{K}_{k+1} \mathbf{H}_{k+1} \mathbf{P}_{k+1}^{-}
\end{aligned}
$$

where $\mathbf{H}_{k}=\partial \mathbf{h}_{k} / \partial \mathbf{x}_{k}$ is the measurement partial computed at $t_{k}$ and $\mathbf{z}_{k+1}^{*}$ is the actual observation and the difference between the actual and predicted measurement (i.e., $\mathbf{z}_{k+1}^{*}-\mathbf{n}_{k+1}^{-}$) is called the residual or innovation. 
Among many important properties of the EKF, we point out the two which will be discussed later in more details. Considering the gain Eqn. (42) and the mean update Eqn. (43), we observe that as the $a$ priori covariance matrix becomes more accurate (i.e., $\mathbf{P}_{k+1}^{-} \rightarrow \mathbf{0}$ ) the filter values the residual less (i.e., the actual measurement is trusted less). On the other hand, as the measurement becomes more accurate (i.e., $\mathbf{R}_{k+1} \rightarrow \mathbf{0}$ ) the filter values the residual more (i.e., the actual measurement is trusted more). Therefore, optimally weighting the residual is a critical component of maximizing the filter performance.

\section{Higher Order Semi-Analytic Extended Kalman Filter}

In deriving of the higher order semi-analytic EKF (HAEKF), we assume that the trajectory solutions (i.e., STTs) are computed over some time span prior to filtering. Under this assumption the local trajectory motion can be mapped analytically while incorporating nonlinear effects and the same analogy applies when mapping the trajectory statistics.

For any dynamical systems, the current state mean and covariance matrix can be mapped as functions of the epoch state distribution. Using the STT notation ${ }^{10,11,39,40}$

$$
\begin{aligned}
\delta \mathbf{m}_{k+1}^{i}\left(\delta \mathbf{x}_{k+1}\right) & =E\left[\delta \mathbf{x}_{k+1}^{i}\right] \\
& =\sum_{p=1}^{m} \frac{1}{p !} \phi_{\left(t_{k+1}, t_{k}\right)}^{i, \gamma_{1} \cdots \gamma_{p}} E\left[\delta \mathbf{x}_{k}^{\gamma_{1}} \cdots \delta \mathbf{x}_{k}^{\gamma_{p}}\right] \\
\mathbf{P}_{k+1}^{i j}\left(\delta \mathbf{x}_{k+1}\right) & =E\left[\left(\delta \mathbf{x}_{k+1}^{i}-\delta \mathbf{m}_{k+1}^{i}\right)\left(\delta \mathbf{x}_{k+1}^{j}-\delta \mathbf{m}_{k+1}^{j}\right)\right] \\
& =\left(\sum_{p=1}^{m} \sum_{q=1}^{m} \frac{1}{p ! q !} \phi_{\left(t_{k+1}, t_{k}\right)}^{i, \gamma_{1} \cdots \gamma_{p}} \phi_{\left(t_{k+1}, t_{k}\right)}^{j, \zeta_{1} \cdots \zeta_{q}} E\left[\delta \mathbf{x}_{k}^{\gamma_{1}} \cdots \delta \mathbf{x}_{k}^{\gamma_{p}} \delta \mathbf{x}_{k}^{\zeta_{1}} \cdots \delta \mathbf{x}_{k}^{\zeta_{q}}\right]\right)-\delta \mathbf{m}_{k+1}^{i} \delta \mathbf{m}_{k+1}^{j}
\end{aligned}
$$

where $\left\{\gamma_{j}, \zeta_{j}\right\} \in\{1, \cdots, n\}$ and $E\left[\mathbf{x}_{k+1}\right]=\mathbf{m}_{k+1}=\phi\left(t_{k+1} ; \mathbf{x}_{k}, t_{k}\right)+\delta \mathbf{m}_{k+1}$. Now, the only unknowns are the expectations (i.e., moments) of the deviations in Eqns. (45-46). Even if the trajectory is initiated with the Gaussian a priori, except for the case $m=1$, it is obvious that the mapped trajectory distribution is no longer a Gaussian due to system nonlinearity. Hence, exact computation of the higher order moments is a difficult process, if not impossible.

In the particle-based filters, this problem is remedied by using an ensemble of sample points to approximate the pdf. A more formal approach is to use the Edgeworth/Gram-Chalier ${ }^{1}$ or Laplace approximations to compute the higher order moments; however, these approaches are beyond the scope of this paper. In practice, especially in trajectory navigation, however, the Gaussian assumption has proven to provide a sufficiently accurate statistical approximation. Therefore, we assumed that the updated estimates behave as Gaussian and implement the joint characteristic function to compute the higher order moments. Note that, as apparent from Eqn. (46) $2 m$ th-order moments are required for covariance matrix computation. As the order of solution increases, i.e., $m \rightarrow \infty$, the higher order solution will yield the true Monte-Carlo mean and covariance matrix: ${ }^{38}$

$$
\begin{aligned}
\mathbf{m}_{k+1}^{i} & =\frac{1}{N} \sum_{\alpha=1}^{N} \phi_{i}\left(t_{k+1} ; \mathbf{x}_{k}^{\alpha}, t_{k}\right) \\
\mathbf{P}_{k+1}^{i j} & =\frac{1}{N-1} \sum_{\alpha=1}^{N}\left[\phi^{i}\left(t_{k+1} ; \mathbf{x}_{k}^{\alpha}, t_{k}\right)-\mathbf{m}_{k+1}^{i}\right]\left[\phi^{j}\left(t_{k+1} ; \mathbf{x}_{k}^{\alpha}, t_{k}\right)-\mathbf{m}_{k+1}^{j}\right]
\end{aligned}
$$

where the superscript $\alpha$ represents the sample points that are chosen according to a probability distribution at $t_{k}$.

Now suppose at $t_{k}$, a Gaussian distribution sufficiently approximates statistics of the state $\mathbf{x}_{k}$. The moments in Eqn. (46) can then be computed using the joint characteristic functions, i.e., Eqn. (37), and thus, the propagated mean and covariance matrix at $t_{k+1}$ are functions of the first two moments. Note that this prediction step is a simple algebraic operation since we assume the STTs are computed previously. If we consider a zero initial mean, all the odd moments of the initial conditions vanish, which is the unique property of the Gaussian distribution, and the above equations simplify a great deal. Moreover, it is clear from Eqn. (45) that the mean will not be zero indicating that the mean deviates from the reference trajectory, whereas the linear analysis assumes the mean being the reference trajectory. 
By applying the Gaussian assumption, the higher order semi-analytic extended Kalman filter algorithm can be stated as follows:

\section{Prediction:}

$$
\begin{aligned}
\left(\mathbf{m}_{k+1}^{-}\right)^{i} & =E\left[\phi^{i}\left(t_{k+1} ; \mathbf{m}_{k}^{+}+\delta \mathbf{m}_{k}^{+}, t_{k}\right)\right]=\phi^{i}\left(t_{k+1} ; \mathbf{m}_{k}^{+}, t_{k}\right)+\delta \mathbf{m}_{k+1}^{i} \\
& =\phi^{i}\left(t_{k+1} ; \mathbf{m}_{k}^{+}, t_{k}\right)+\sum_{p=1}^{m} \frac{1}{p !} \phi_{\left(t_{k+1}, t_{k}\right)}^{i, \gamma_{1} \cdots \gamma_{p}} E\left[\delta \mathbf{x}_{k}^{\gamma_{1}} \cdots \delta \mathbf{x}_{k}^{\gamma_{p}}\right] \\
\left(\mathbf{P}_{k+1}^{-}\right)^{i j} & =\mathbf{P}_{k+1}^{i j}+\mathbf{Q}_{k}^{i j} \\
& =\left(\sum_{p=1}^{m} \sum_{q=1}^{m} \frac{1}{p ! q !} \phi_{\left(t_{k+1}, t_{k}\right)}^{i, \gamma_{1} \cdots \gamma_{p}} \phi_{\left(t_{k+1}, t_{k}\right)}^{j, \zeta_{1} \cdots \zeta_{q}} E\left[\delta \mathbf{x}_{k}^{\gamma_{1}} \cdots \delta \mathbf{x}_{k}^{\gamma_{p}} \delta \mathbf{x}_{k}^{\zeta_{1}} \cdots \delta \mathbf{x}_{k}^{\zeta_{q}}\right]\right)-\delta \mathbf{m}_{k+1}^{i} \delta \mathbf{m}_{k+1}^{j}+\mathbf{Q}_{k}^{i j} \\
\left(\mathbf{n}_{k+1}^{-}\right)^{i} & =E\left[\left(\mathbf{z}_{k+1}^{-}\right)^{i}\right]=E\left[\mathbf{h}^{i}\left(t_{k+1} ; \mathbf{m}_{k}^{+}+\delta \mathbf{m}_{k}^{+}, t_{k}\right)+\mathbf{v}_{k+1}\right]=\mathbf{h}^{i}\left(t_{k+1} ; \mathbf{m}_{k}^{+}, t_{k}\right)+\delta \mathbf{n}_{k+1}^{i} \\
& =\mathbf{h}^{i}\left(t_{k+1} ; \mathbf{m}_{k}^{+}, t_{k}\right)+\sum_{p=1}^{m} \frac{1}{p !} \mathbf{h}_{\left(t_{k+1}, t_{k}\right)}^{i, \gamma_{1} \cdots \gamma_{p}} E\left[\delta \mathbf{x}_{k}^{\gamma_{1}} \cdots \delta \mathbf{x}_{k}^{\gamma_{p}}\right]
\end{aligned}
$$

where

$$
\mathbf{h}_{\left(t_{k+1}, t_{k}\right)}^{i, \gamma_{1} \cdots \gamma_{p}}=\frac{\partial^{p} \mathbf{h}^{i}}{\partial \mathbf{x}_{k}^{\gamma_{1}} \cdots \partial \mathbf{x}_{k}^{\gamma_{p}}}
$$

Update:

$$
\begin{aligned}
\left(\mathbf{P}_{k+1}^{\mathbf{z z}}\right)^{i j} & =E\left[\left(\mathbf{z}_{k+1}^{-}-\mathbf{n}_{k+1}^{-}\right)^{i}\left(\mathbf{z}_{k+1}^{-}-\mathbf{n}_{k+1}^{-}\right)^{j}\right]=E\left[\left(\mathbf{z}_{k+1}^{-}\right)^{i}\left(\mathbf{z}_{k+1}^{-}\right)^{j}\right]-\left(\mathbf{n}_{k+1}^{-}\right)^{i}\left(\mathbf{n}_{k+1}^{-}\right)^{j} \\
& =E\left[\left(\mathbf{h}\left(t_{k+1} ; \mathbf{m}_{k}^{+}+\delta \mathbf{m}_{k}^{+}, t_{k}\right)+\mathbf{v}_{k+1}\right)^{i}\left(\mathbf{h}\left(t_{k+1} ; \mathbf{m}_{k}^{+}+\delta \mathbf{m}_{k}^{+}, t_{k}\right)+\mathbf{v}_{k+1}\right)^{j}\right]-\left(\mathbf{n}_{k+1}^{-}\right)^{i}\left(\mathbf{n}_{k+1}^{-}\right)^{j} \\
& =\left(\mathbf{R}_{k+1}^{i j}+\sum_{p=1}^{m} \sum_{q=1}^{m} \frac{1}{p ! q !} \mathbf{h}_{\left(t_{k+1}, t_{k}\right)}^{i, \gamma_{1} \cdots \gamma_{p}} \mathbf{h}_{\left(t_{k+1}, t_{k}\right)}^{j, \zeta_{1} \cdots \zeta_{q}} E\left[\delta \mathbf{x}_{k}^{\gamma_{1}} \cdots \delta \mathbf{x}_{k}^{\gamma_{p}} \delta \mathbf{x}_{k}^{\zeta_{1}} \cdots \delta \mathbf{x}_{k}^{\zeta_{q}}\right]\right)-\left(\delta \mathbf{n}_{k+1}^{-}\right)^{i}\left(\delta \mathbf{n}_{k+1}^{-}\right)^{j} \\
\left(\mathbf{P}_{k+1}^{\mathbf{x z}}\right)^{i j} & =E\left[\left(\mathbf{x}_{k+1}^{-}-\mathbf{m}_{k+1}^{-}\right)^{i}\left(\mathbf{z}_{k+1}^{-}-\mathbf{n}_{k+1}^{-}\right)^{j}\right]=E\left[\left(\mathbf{x}_{k+1}^{-}\right)^{i}\left(\mathbf{z}_{k+1}^{-}\right)^{j}\right]-\left(\mathbf{m}_{k+1}^{-}\right)^{i}\left(\mathbf{n}_{k+1}^{-}\right)^{j} \\
& =E\left[\left(\phi\left(t_{k+1} ; \mathbf{m}_{k}^{+}+\delta \mathbf{m}_{k}^{+}, t_{k}\right)\right)^{i}\left(\mathbf{h}\left(t_{k+1} ; \mathbf{m}_{k}^{+}+\delta \mathbf{m}_{k}^{+}, t_{k}\right)+\mathbf{v}_{k+1}\right)^{j}\right]-\left(\mathbf{m}_{k+1}^{-}\right)^{i}\left(\mathbf{n}_{k+1}^{-}\right)^{j} \\
& =\left(\sum_{p=1}^{m} \sum_{q=1}^{m} \frac{1}{p ! q !} \phi_{\left(t_{k+1}, t_{k}\right)}^{i, \zeta_{1} \cdots \zeta_{q}} \mathbf{h}_{\left(t_{k+1}, \gamma_{k}\right)}^{j, \gamma_{1} \cdots \gamma_{p}} E\left[\delta \mathbf{x}_{k}^{\gamma_{1}} \cdots \delta \mathbf{x}_{k}^{\gamma_{p}} \delta \mathbf{x}_{k}^{\zeta_{1}} \cdots \delta \mathbf{x}_{k}^{\zeta_{q}}\right]\right)-\left(\delta \mathbf{m}_{k+1}^{-}\right)^{i}\left(\delta \mathbf{n}_{k+1}^{-}\right)^{j} \\
\mathbf{K}_{k+1} & =\mathbf{P}_{k+1}^{\mathbf{x z}}\left(\mathbf{P}_{k+1}^{\mathbf{z z}}\right)^{-1} \\
\mathbf{m}_{k+1}^{+} & =\mathbf{m}_{k+1}^{-}+\mathbf{K}_{k+1}\left(\mathbf{z}_{k+1}^{*}-\mathbf{n}_{k+1}^{-}\right) \\
\mathbf{P}_{k+1}^{+} & =\mathbf{P}_{k+1}^{-}-\mathbf{K}_{k+1} \mathbf{P}_{k+1}^{\mathbf{z z}} \mathbf{K}_{k+1}^{T}
\end{aligned}
$$


Note that when the measurement function Eqn. (30) is linear in $\mathbf{x}_{k}$, Eqn. (51) simplifies to

$$
\begin{aligned}
& \left(\mathbf{n}_{k+1}^{-}\right)^{i}=\mathbf{h}^{i}\left(t_{k+1} ; \mathbf{m}_{k}^{+}, t_{k}\right)+\mathbf{h}_{k+1}^{i, \alpha} \sum_{p=1}^{m} \frac{1}{p !} \phi_{\left(t_{k+1}, t_{k}\right)}^{\alpha, \gamma_{1} \cdots \gamma_{p}} E\left[\delta \mathbf{x}_{k}^{\gamma_{1}} \cdots \delta \mathbf{x}_{k}^{\gamma_{p}}\right]=\mathbf{h}^{i}\left(\mathbf{m}_{k+1}^{-}, t_{k+1}\right) \\
& \left(\mathbf{P}_{k+1}^{\mathbf{z z}}\right)^{i j}=\left(\mathbf{R}_{k+1}^{i j}+\mathbf{h}_{k+1}^{i, \alpha} \mathbf{h}_{k+1}^{j, \beta} \sum_{p=1}^{m} \sum_{q=1}^{m} \frac{1}{p ! q !} \phi_{\left(t_{k+1}, t_{k}\right)}^{\alpha, \gamma_{1} \cdots \gamma_{p}} \phi_{\left(t_{k+1}, t_{k}\right)}^{\beta, \zeta_{1} \cdots \zeta_{q}} E\left[\delta \mathbf{x}_{k}^{\gamma_{1}} \cdots \delta \mathbf{x}_{k}^{\gamma_{p}} \delta \mathbf{x}_{k}^{\zeta_{1}} \cdots \delta \mathbf{x}_{k}^{\zeta_{q}}\right]\right) \\
& -\left(\delta \mathbf{n}_{k+1}^{-}\right)^{i}\left(\delta \mathbf{n}_{k+1}^{-}\right)^{j} \\
& =\left(\mathbf{R}_{k+1}^{i j}+\mathbf{h}_{k+1}^{i, \alpha} \mathbf{h}_{k+1}^{j, \beta}\left(\mathbf{P}_{k+1}^{-}\right)^{\alpha \beta}\right)-\left(\delta \mathbf{n}_{k+1}^{-}\right)^{i}\left(\delta \mathbf{n}_{k+1}^{-}\right)^{j} \\
& =\left(\mathbf{H}_{k+1} \mathbf{P}_{k+1}^{-} \mathbf{H}_{k+1}^{T}+\mathbf{R}_{k+1}\right)^{i j} \\
& \left(\mathbf{P}_{k+1}^{\mathbf{x z}}\right)^{i j}=\left(\mathbf{h}_{k+1}^{j, \alpha} \sum_{p=1}^{m} \sum_{q=1}^{m} \frac{1}{p ! q !} \phi_{\left(t_{k+1}, t_{k}\right)}^{i, \gamma_{1} \cdots \gamma_{p}} \phi_{\left(t_{k+1}, t_{k}\right)}^{\alpha, \zeta_{1} \cdots \zeta_{q}} E\left[\delta \mathbf{x}_{k}^{\gamma_{1}} \cdots \delta \mathbf{x}_{k}^{\gamma_{p}} \delta \mathbf{x}_{k}^{\zeta_{1}} \cdots \delta \mathbf{x}_{k}^{\zeta_{q}}\right]\right)-\left(\delta \mathbf{m}_{k+1}^{-}\right)^{i}\left(\delta \mathbf{n}_{k+1}^{-}\right)^{j} \\
& =\left(\left(\mathbf{P}_{k+1}^{-}\right)^{i \alpha} \mathbf{h}_{k+1}^{j, \alpha}\right)-\left(\delta \mathbf{m}_{k+1}^{-}\right)^{i}\left(\delta \mathbf{n}_{k+1}^{-}\right)^{j} \\
& =\left(\mathbf{P}_{k+1}^{-} \mathbf{H}_{k+1}^{T}\right)^{i j}
\end{aligned}
$$

which indicates that the measurement prediction and update equations are the same as in the EKF algorithm.

Also, note that when $m=1$, the higher order semi-analytic filter becomes the linear Kalman filter (LKF), not the EKF. The superiority of the EKF over the LKF is clearly demonstrated in Maybeck. ${ }^{1}$ However, when the reference trajectory is relatively close to the true trajectory, the HAEKF can provide a more accurate solution than the EKF.

\section{Higher Order Numerical Extended Kalman Filter}

From the derivation of the HAEKF, it is obvious that we can also derive a higher order numerical EKF (HNEKF) by directly integrating the STTs for each time interval between the measurements. The filter algorithm is identical to the HAEKF except that the trajectory and STTs are integrated according to Eqns. (12-15). We note that this process is numerically quite intensive as we consider higher order solutions; however, the HNEKF can yield the most accurate solution. If we consider the case $m=1$, the HNEKF becomes the EKF shown in Eqns.(39-44).

\section{E. Unscented Kalman Filter}

The unscented Kalman filter, first introduced by Julier and Uhlmann, ${ }^{14,15,18}$ is being implemented in a diverse field of engineering, science, and economics due to its simplicity while providing faster convergence and better accuracy than the extended Kalman filter. The UKF is initialized with the following pre-determined sigma points:

$$
\begin{aligned}
\mathcal{X}_{k}^{0} & =\mathbf{m}_{k}^{+} \\
\mathcal{W}_{k}^{0} & =\kappa /(n+\kappa) \\
\mathcal{X}_{k}^{i} & =\mathbf{m}_{k}^{+}+\left[\sqrt{(n+\kappa) \mathbf{P}_{k}}\right]_{i} \\
\mathcal{W}_{k}^{i} & =1 /[2(n+\kappa)] \\
\mathcal{X}_{k}^{i+n} & =\mathbf{m}_{k}^{+}-\left[\sqrt{(n+\kappa) \mathbf{P}_{k}}\right]_{i} \\
\mathcal{W}_{k}^{i+n} & =1 /[2(n+\kappa)]
\end{aligned}
$$

where $\kappa \in \Re, \mathcal{X}_{k}^{j}$ are the sample points with associated weights $\mathcal{W}_{k}^{j}$, and $\left[\sqrt{(n+\kappa) \mathbf{P}\left(t_{0}\right)}\right]_{i}$ are the $i$ th row of the matrix square root of $\left[(n+\kappa) \mathbf{P}\left(t_{0}\right)\right]$.

The unscented Kalman filter algorithm for additive (linear) process and measurement noises is as follows: 
Prediction:

$$
\begin{aligned}
\mathcal{X}_{k+1}^{i} & =\phi\left(t_{k+1} ; \mathcal{X}_{k}^{i}, t_{k}\right) \\
\mathbf{m}_{k+1}^{-} & =\sum_{i=0}^{2 n} \mathcal{W}_{k}^{i} \mathcal{X}_{k+1}^{i} \\
\mathbf{P}_{k+1}^{-} & =\sum_{i=0}^{2 n} \mathcal{W}_{k}^{i}\left[\mathcal{X}_{k+1}^{i}-\mathbf{m}_{k+1}^{-}\right]\left[\mathcal{X}_{k+1}^{i}-\mathbf{m}_{k+1}^{-}\right]^{T}+\mathbf{Q}_{k} \\
\mathcal{Z}_{k+1}^{i} & =\mathbf{h}\left(\mathcal{X}_{k+1}^{i}\right) \\
\mathbf{n}_{k+1}^{-} & =\sum_{i=0}^{2 n} \mathcal{W}_{k}^{i} \mathcal{Z}_{k+1}^{i}
\end{aligned}
$$

Update:

$$
\begin{aligned}
\mathbf{P}_{k+1}^{\mathbf{z z}} & =\sum_{i=0}^{2 n} \mathcal{W}_{k}^{i}\left[\mathcal{Z}_{k+1}^{i}-\mathbf{n}_{k+1}^{-}\right]\left[\mathcal{Z}_{k+1}^{i}-\mathbf{n}_{k+1}^{-}\right]^{T}+\mathbf{R}_{k} \\
\mathbf{P}_{k+1}^{\mathbf{x z}} & =\sum_{i=0}^{2 n} \mathcal{W}_{k}^{i}\left[\mathcal{X}_{k+1}^{i}-\mathbf{m}_{k+1}^{-}\right]\left[\mathcal{Z}_{k+1}^{i}-\mathbf{n}_{k+1}^{-}\right]^{T} \\
\mathbf{m}_{k+1}^{+} & =\mathbf{m}_{k+1}^{-}+\mathbf{K}_{k+1}\left(\mathbf{z}_{k+1}^{*}-\mathbf{n}_{k+1}^{-}\right) \\
\mathbf{P}_{k+1}^{+} & =\mathbf{P}_{k+1}^{-}-\mathbf{K}_{k+1} \mathbf{P}_{k+1}^{\mathbf{z z}} \mathbf{K}_{k+1}^{T} \\
\mathbf{K}_{k+1} & =\mathbf{P}_{k+1}^{\mathbf{x z}}\left(\mathbf{P}_{k+1}^{\mathbf{z z}}\right)^{-1}
\end{aligned}
$$

\section{Examples}

In this section, we present several simulations of a halo orbit, which is a Lissajous type periodic orbit where the in-plane and out-of-plane frequencies are the same, computed based on the circular restricted three-body problem (CR3BP). The governing equations of motion for CR3BP, in non-dimensional form, are given $\mathrm{as}^{41}$

$$
\begin{aligned}
\ddot{x}-2 \dot{y} & =\frac{\partial U}{\partial x} \\
\ddot{y}+2 \dot{x} & =\frac{\partial U}{\partial y} \\
\ddot{z} & =\frac{\partial U}{\partial z}
\end{aligned}
$$

where

$$
\begin{aligned}
U & =\frac{(1-\mu)}{r_{1}}+\frac{\mu}{r_{2}}+\frac{\left(x^{2}+y^{2}\right)}{2} \\
r_{1} & =\left[(x+\mu)^{2}+y^{2}+z^{2}\right]^{1 / 2} \\
r_{2} & =\left[(x-1+\mu)^{2}+y^{2}+z^{2}\right]^{1 / 2} .
\end{aligned}
$$

Here, $U$ is the CR3BP potential, $(x, y, z)$ are the spacecraft position components, $(u, v, w)$ are the spacecraft velocity components, and $\mu=\mu_{\oplus} /\left(\mu_{S}+\mu_{\oplus}\right)$, where $\mu_{S}$ is the solar gravitational constant $(1.32712440018 \times$ $\left.10^{11} \mathrm{~km}^{3} / \mathrm{s}^{2}\right)$ and $\mu_{\oplus}$ is the Earth gravitational constant $\left(398600.44 \mathrm{~km}^{3} / \mathrm{s}^{2}\right)$.

The units can be dimensionalized by applying the length scale of $\ell=1 \mathrm{AU}=1.49597870691 \times 10^{8} \mathrm{~km}$, where AU stands for astronomical unit, and time scale of $\tau=1 / \omega_{E}$, where $\omega_{E}$ is the mean motion of the Earth about the Sun (i.e., $\sqrt{A U^{3} / \mu_{S}}$ ). There exist many techniques to efficiently compute halo orbits, and we have implemented a third order analytic solution as the initial guess and applied differential corrections to obtain convergence to the true halo orbit solution. ${ }^{42,43}$ Figure 1 shows the reference (nominal) trajectory for one orbital period ( $\sim 177.86$ days), which corresponds to the case 1 given in Table 1 . 
Table 1. Halo orbit maximum amplitudes with respect to the $L_{1}$ point

\begin{tabular}{cccc}
\hline \hline Cases & $A_{x}(\mathrm{~km})$ & $A_{y}(\mathrm{~km})$ & $A_{z}(\mathrm{~km})$ \\
\hline 1 & 245924 & 668228 & 137908 \\
2 & 246069 & 668416 & 139015 \\
\hline \hline
\end{tabular}

For the measurement model, we assume a simple linear model where only the $y$-coordinate is observed, i.e.,

$$
\mathbf{z}_{k+1}=y_{k+1}+\mathbf{v}_{k+1}
$$

This can be viewed as a range measurement obtained by optical imaging of the Earth relative to distant stars. The measurement noise is assumed to be $0.1 \mathrm{~m}$ for each range measurement. The linear assumption simplifies the problem a great deal since the measurement sensitivity does not require the computation of the higher order partials. This way, it is easier to understand the effect of the nonlinear orbit uncertainty propagation on the filter performance.

At epoch the initial state is assumed to be a zero mean Gaussian with position uncertainties of 100 $\mathrm{km}$ and velocity uncertainties of $0.1 \mathrm{~m} / \mathrm{s}$. The initial mean and covariance matrix are mapped using the STT approach for $m=\{1,3\}$, unscented transformation, and Monte-Carlo simulations based on $10^{6}$ sample points, and Figure 2 shows the mean and the projection of the covariance matrix onto the $x-y$ plane after one orbital period. Assuming the MC simulation is the true solution, the result shows that the 3rd order solution is the most accurate approximation, whereas the linear solution fails to characterize the orbit uncertainty distribution.

We now consider the same initial uncertainties, but assume the initial guess (mean) is off by $100 \mathrm{~km}$ for the position components and $0.1 \mathrm{~m} / \mathrm{s}$ for the velocity components. A set of pseudo-measurements are computed based on the reference trajectory with a 20-day increment. Using the same measurements, the initial mean and covariance matrix are mapped and solved using the EKF, UKF, 3rd order HNEKF, and 3rd order HAEKF. For HAEKF, since the trajectory is periodic, the STTs are computed and stored for one orbital period, which is divided into two segments for numerical consistency. Figure 3 shows the a priori and a posteriori position and velocity uncertainties, where $\sigma_{R}=\sqrt{\sigma_{x x}+\sigma_{y y}+\sigma_{z z}}$ and $\sigma_{V}=\sqrt{\sigma_{u u}+\sigma_{v v}+\sigma_{w w}}$. A sudden drop in the uncertainties right after 100 days is due to the fact that the initial covariance matrix is quite large and it requires at least six independent measurements to obtain a well-defined (i.e., reduced to the measurement noise level in all directions) a posteriori covariance matrix. The result shows that the EKF overestimates the uncertainties (i.e., assumes they are smaller than they are in actuality) while the UKF, HNEKF, and HAEKF provide conservative uncertainty estimates. Figure 4 shows the magnitude of the absolute position and velocity errors, i.e., the difference between the updated mean and the true state. The result shows that the EKF does not perform well as compared to the higher order filters. This clearly explains the importance of nonlinear orbit uncertainty propagation. The covariance matrix computed by using the first order method (i.e., EKF) overestimates the solution, and hence, the residual is less trusted. On the other hand, the UKF and the higher order filters predict more conservative uncertainties and more effectively balance the a priori uncertainties and the actual measurements (i.e., measurements are valued more than the a priori information in this case). Figures 5 and 6 are based on the same filter setup except that the measurements are updated every 5 days. It shows that there is not much difference in the propagated uncertainties, but the absolute errors are computed more accurately in UKF and higher order filter runs.

Figures 7 and 8 show the HNEKF results for cases $m \in\{1,2,3\}$. As mentioned earlier, note that the case $m=1$ is identical to the EKF formulation. The result shows that the higher order filters, $m \in\{2,3\}$, provide superior filter performance over the first order case and it is observed that the second order effect contains most of the system nonlinearity, indicating that the second order filter is sufficient for an accurate nonlinear filter in our example.

Figure 9 shows the HAEKF absolute error plots for $m \in\{1,2,3\}$. The uncertainties for $m=1$ are similar to the EKF solution and for $m=2$ are similar to the case $m=3$ as shown in Figure 3 . The absolute error plot shows that all three filters provide good estimation performance even for the case $m=1$. This is expected since the pseudo-measurements are computed based on the reference trajectory which the STTs are computed based on. In other words, the reference trajectory can be thought of as a regression solution for the simulated measurements. In order to analyze the higher order effect, the pseudo-measurements are 
generated from the case 2 halo orbit given in Table 1. Figures 10 and 11 show the simulated filter solutions. The results show that the higher order solutions are superior over the linear filters, i.e., EKF and HAEKF for $m=1$. As expected, this indicates that the linear Kalman filter is only feasible when the reference trajectory is sufficiently close to the true trajectory. The HAEKFs for $m>1$, however, have more flexibility in the reference trajectory. The overall filter convergence is slightly slower than the previous cases since the initial mean is assumed to be the same as in the previous cases, and thus, it is farther away from the true trajectory (i.e., the trajectory which the pseudo-measurements are generated).

\section{A. Discussion}

In this study, the EKF required integration of $n+n^{2}=42$ equations and the UKF required integration of $(2 n+1) n=78$ equations between each measurement update, and in the actual filter runs, the EKF was slightly faster than the UKF. The HNEKFs for $m>1$ provide superior results over the linear filters (even UKF when $m>2$ ); however, the computational load increases significantly as $m$ increases. For example, the third order HNEKF requires integration of 1554 equations. On the other hand, the HAEKF does not require any integration in the the actual filtering process. The most expensive numerical operation in the HAEKF is the higher order moment computation; however, there exist various techniques for efficient computation of moments. Hence, for the missions with pre-determined reference trajectories, the higher order semi-analytic filter may be suitable for the trajectory navigation while obtaining faster convergence and a more accurate filter solution than the EKF.

\section{Conclusion}

In this study, we have presented research showing the importance of nonlinear orbit uncertainty propagation for spacecraft trajectory navigation. Four different filtering methods, extended Kalman filter, unscented Kalman filter, higher order numerical extended Kalman filter, and higher order semi-analytic extended Kalman filter, were compared based on a halo orbit about the Sun-Earth $L_{1}$ point with realistic measurement accuracies. The results showed that a higher order filter provides a faster convergence and a superior filter solution over linear filters. Also, the Gaussian assumption of the a posteriori state yielded a sufficient approximation even for nonlinear filters. For the cases where the reference trajectory was relatively close to the true trajectory, the higher order semi-analytic filter provided solutions essentially equivalent to both the UKF and HNEKF, and yielded a much faster filter process. This indicates that once trajectory solutions are stored on a spacecraft, an auto-navigation processor that incorporates trajectory nonlinearity and allows fast convergence may be feasible in practice.

\section{Acknowledgement}

The research described in this paper was sponsored by a grant from the Jet Propulsion Laboratory, California Institute of Technology which is under contract with the National Aeronautics and Space Administration.

\section{References}

${ }^{1}$ Maybeck, P., Stochastic Models, Estimation, and Control. Vol 2, Academic Press, 1982, pp. 159-271.

${ }^{2}$ Montenbruck, O. and Gill, E., Satellite Orbits, Springer, 2nd ed., 2001, pp. 257-291.

${ }^{3}$ Crassidis, J. and Junkins, J., Optimal Estimation of Dynamics Systems, CRC Press LLC, 2004, pp. 243-410.

${ }^{4}$ Anderson, J., Colombo, G., Friedman, L., and Lau, E., An Arrow to the Sun: In Gravitazione Sperimentale, Rome: Accademia Nazionale dei Lincei, 1977, 393-422.

${ }^{5}$ Mease, K., Anderson, J., Wood, L., and White, L., "Tests of General Relativity Using Starprobe Radio Metric Tracking Data," Vol. 7, No. 1, 1983, pp. 36-44.

${ }^{6}$ Bertotti, B., Iess, L., and Tortora, P., "A Test of General Relativity using Radio Links with the Cassini Spacecraft," Nature, Vol. 425, 2003, pp. 374-376.

${ }^{7}$ Scheeres, D., Han, D., and Hou, Y., "Influence of Unstable Manifolds on Orbit Uncertainty," Journal of Guidance, Control, and Dynamics, Vol. 24, No. 3, 2001, pp. 573-585.

${ }^{8}$ Longuski, J., Fischbach, E., Scheeres, D., Giampieri, G., and Park, R., "Deflection of Spacecraft Trajectories as a New Test of General Relativity: Determining the PPN Parameters $\beta$ and $\gamma$," Phys. Rev. D, Vol. 69, No. 042001, 2004, pp. 0420011-042001-15. 
${ }^{9}$ Park, R., Scheeres, D., Giampieri, G., Longuski, J., and Fischbach, E., "A Test of General Relativity: Estimating the Parameterized Post-Newtonian Parameters from Spacecraft Radiometric Measurements," Journal of Spacecraft and Rockets, Vol. 42, No. 3, 2005, pp. 559-568.

${ }^{10}$ Park, R. and Scheeres, D., "Nonlinear Mapping of Gaussian Statistics: Theory and Applications to Spacecraft Trajetory Design," accepted for publication in the Journal of Guidance, Control, and Dynamics, March 2006.

${ }^{11}$ Park, R. and Scheeres, D., "Nonlinear Mapping of Gaussian State Uncertainties: Theory and Application to Spacecraft Control and Navigation," Astrodynamics Specialist Conference, August 2005, Lake Tahoe, California, AAS 05-404.

${ }^{12}$ Renault, C. and Scheeres, D., "Optimal Placement of Statistical Maneuvers in an Unstable Orbital Environment," Journal of Guidance, Control, and Dynamics, Vol. 26, No. 5, 2003, pp. 758-769.

${ }^{13}$ Milani, A., Chesley, S., Chodas, P., and Valsecchi, G., Asteroid Close Approaches: Analysis and Potential Impact Detection in Asteroids III, University of Arizona Press, 2002.

${ }^{14}$ Julier, S., Uhlmann, J., and Durrant-Whyte, H., "A New Approach for Filtering Nonlinear Systems," American Control Conference, June 1995, Seattle, WA, pp. 1628-1632.

${ }^{15}$ Julier, S., Uhlmann, J., and Durrant-Whyte, H., "A New Method For the Nonlinear Transformation of Means and Covariances in Filters and Estimators," IEEE Transactions on Automatic Control, Vol. 45, No. 3, March 2000 , pp. 477-482.

${ }^{16}$ Julier, S. and Uhlmann, J., "The scaled unscented transformation," American Control Conference, 2002, Anchorage, AK, pp. $4555-4559$

${ }^{17}$ Julier, S. and Uhlmann, J., "Unscented Filtering and Nonlinear Estimation," Proceedings of the IEEE, Vol. 92, No. 3, March 2004, pp. 401-422.

${ }^{18}$ Wan, E. and van der Merwe, R., Kalman Filtering and Neural Networks: The Unscented Kalman Filter, chap. 7 , Wiley, 2001.

${ }^{19}$ Kalman, R., "A New Approach to Linear Filtering and Prediction Problems," Transactions of the ASME, Journal of Basic Engineering, Series D, Vol. 82, March 1960.

${ }^{20}$ Kalman, R. and Bucy, R., "New Results in Linear Filtering and Prediction Theory," Transactions of the ASME, Journal of Basic Engineering, Series D, Vol. 83, Mach 1961.

${ }^{21}$ Azimi-Sadjadi, B. and Krishnaprasad, P., "Approximate Nonlinear Filtering and its Application in Navigation," Automatica, Vol. 41, 2005, pp. 945-956.

${ }^{22}$ Bølviken, E., Acklam, P., Christophersen, N., and Størdal, J.-M., "Monte Carlo Filters for Non-Linear State Estimation," Automatica, Vol. 37, 2001, pp. 177-183.

${ }^{23}$ Gustafsson, F., Gunnarsson, F., Bergman, N., Jansson, U. F. J., Karlsson, R., and Nordlund, P.-J., "Particle Filters for Positioning, Navigation, and Tracking," IEEE Transactions on Signal Processing, Vol. 50, No. 2, Feb. 2002 , pp. $425-437$.

${ }^{24}$ van der Merwe, R., Doucet, A., de Freitas, N., and Wan, E., "The Unscented Particle Filter," Technical Report CUED/FINFENG/TR, August 2000.

${ }^{25}$ Ito, K. and Xiong, K., "Gaussian Filters for Nonlinaer Filtering Problems," IEEE Transactions on Automatic Control, Vol. 45, No. 5, May 2000, pp. 910-927.

${ }^{26}$ Trailović, L., "Variance Estimation and Ranking of Target Tracking Position Error Modeled using Gaussian Mixture Distributions," Automatica, Vol. 41, 2005.

${ }^{27}$ Nørgaard, M., Poulsen, N., and Ravn, O., "New Developments in State Estimation for Nonlinear Systems," Automatica, Vol. 36, No. 11, November 2000, pp. 1627-1638.

${ }^{28}$ Beneš, V., "Approximations to optimal nonlinear filters," IEEE Transactions on Automatic Control, Vol. $12,1967$.

${ }^{29}$ Beneš, V., "New Exact Nonlinear Filters with Large Lie Algebras," Systems and Control Letters, Vol. 5, No. 4, February 1985, pp. 217-221.

${ }^{30}$ Beneš, V., "Exact Finite-Dimensional Filters for Certain Diffusions with Nonlinear Drift," Stochastics, Vol. 5, 1981.

${ }^{31}$ Daum, F., "Exact Finite Dimensional Nonlinear Filters for Continuous Time Processes with Discrete Time Measurements," Proceedings of the 23rd IEEE Conference on Decision and Control, December 1984.

${ }^{32}$ Daum, F., "Exact Finite-Dimensional Nonlinear Filters," IEEE Transactions on Automatic Control, Vol. AC-31, No. 7, July 1986 , pp. 616-622.

${ }^{33}$ Schmidt, G., "Designing Nonlinear Filters based on Daum's Theory," Journal of Guidance, Control, and Dynamics, Vol. 16, No. 2, March-April 1993, pp. 371-376.

${ }^{34}$ Athans, M., Wishner, R., and Bertolini, A., "Suboptimal State Estimation for Continuous-Time Nonlinear Systems from Discrete Noisy Measurements," IEEE Transactions on Automatic Control, Vol. AC-13, No. 5, October 1968, pp. 504-514.

${ }^{35}$ Moulton, F., Differential Equations, The MacMillan Company, 1930.

${ }^{36}$ Soong, T. and Paul, N., "A Second- and Higher Order Perturbation Analysis of Two-Body Trajectories," AIAA Journal, Vol. 9, No. 4, 1971.

${ }^{37}$ Maybeck, P., Stochastic Models, Estimation, and Control. Vol 1, Republished by Navtech Book \& Software Store, 1994, pp. 59-132.

${ }^{38}$ Lass, H. and Gottlieb, P., Probability and Statistics, Addison-Wesley, 1971, pp. 201-229.

${ }^{39}$ Park, R. and Scheeres, D., "Nonlinear Mapping of Gaussian State Uncertainties," JAXA Astrodynamics and Flight Mechanics Meeting, July 2005, Kanagawa, Japan.

${ }^{40}$ Park, R. and Scheeres, D., "Nonlinear Mapping of Gaussian State Covariance and Orbit Uncertainties," Space Flight Mechanics Meeting, January 2005, Copper Mountain, Colorado, AAS 05-170.

${ }^{41}$ Pollard, H., Celestial Mechanics, The Mathematical Association of America, 1976.

${ }^{42}$ Richardson, D., "Analytic Construction of Periodic Orbits About the Collinear Points," Celestial Mechanics, Vol. 22, 1980, pp. 241-253.

${ }^{43}$ Richardson, D. and Cary, N., "A Uniformly Valid Solution for Motion About the Interior Libration Point of the Perturbed Elliptic-Restricted Problem," Astrodynamics Specialist Conference, July 1975, Nassau, Bahamas, AAS 75-021. 

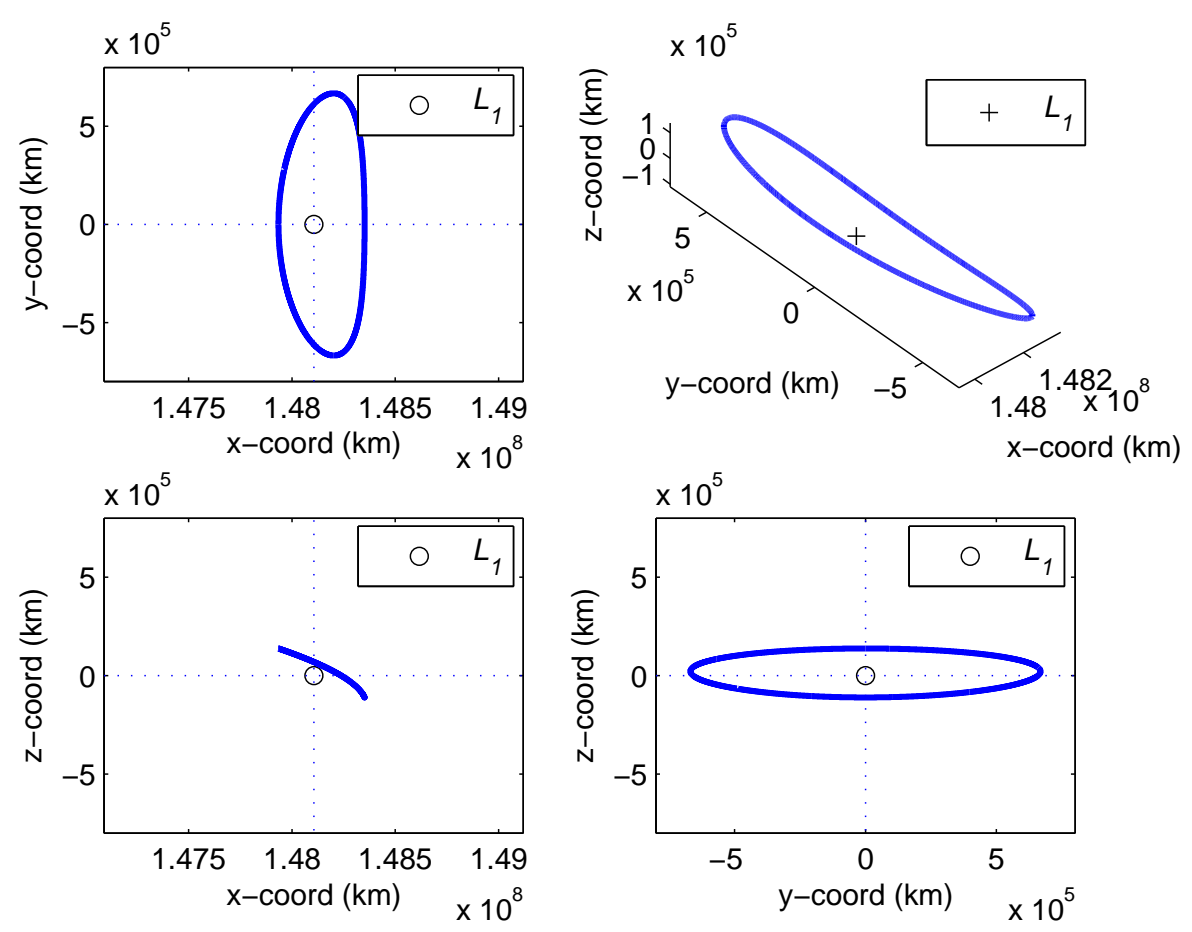

Figure 1. Nominal halo orbit about the Sun-Earth $L_{1}$ point.

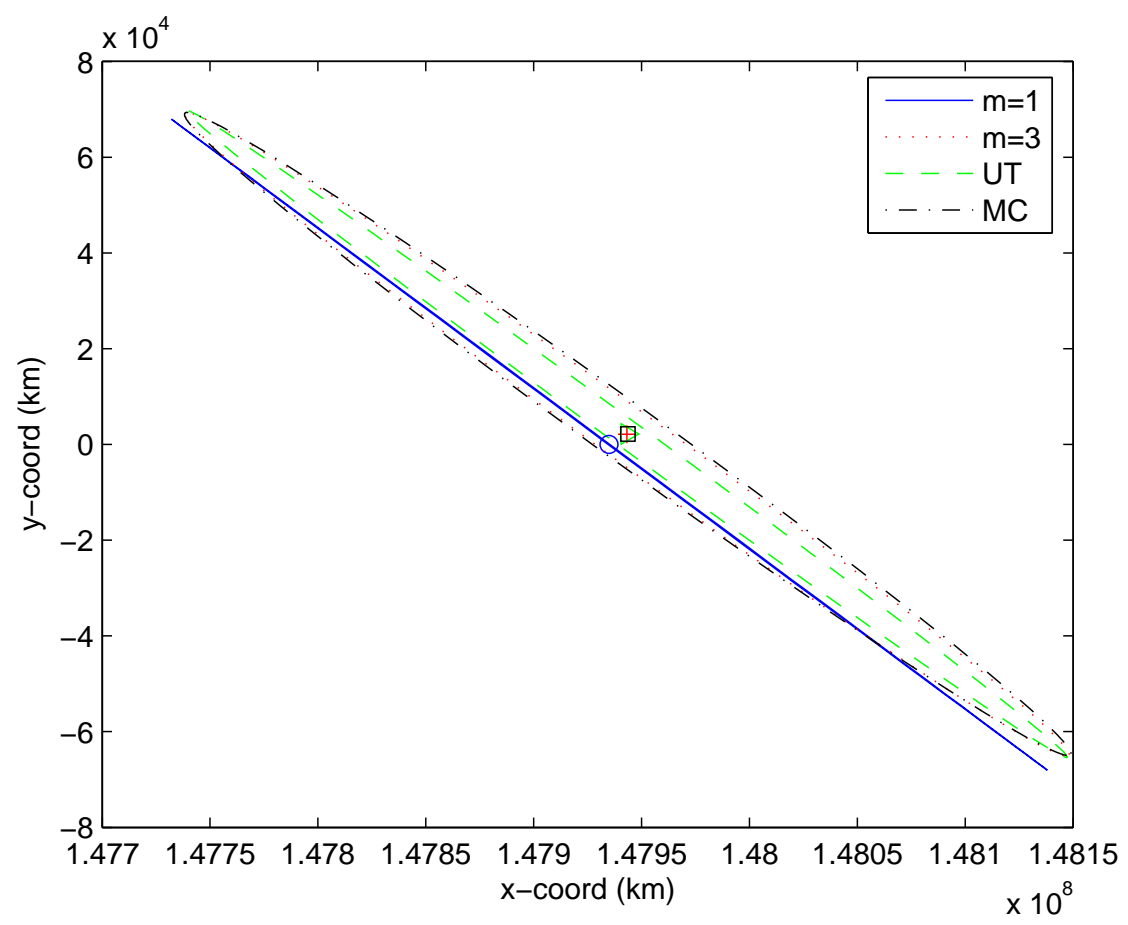

Figure 2. Covariance matrix computed after one orbital period and projected onto the x-y plane. 

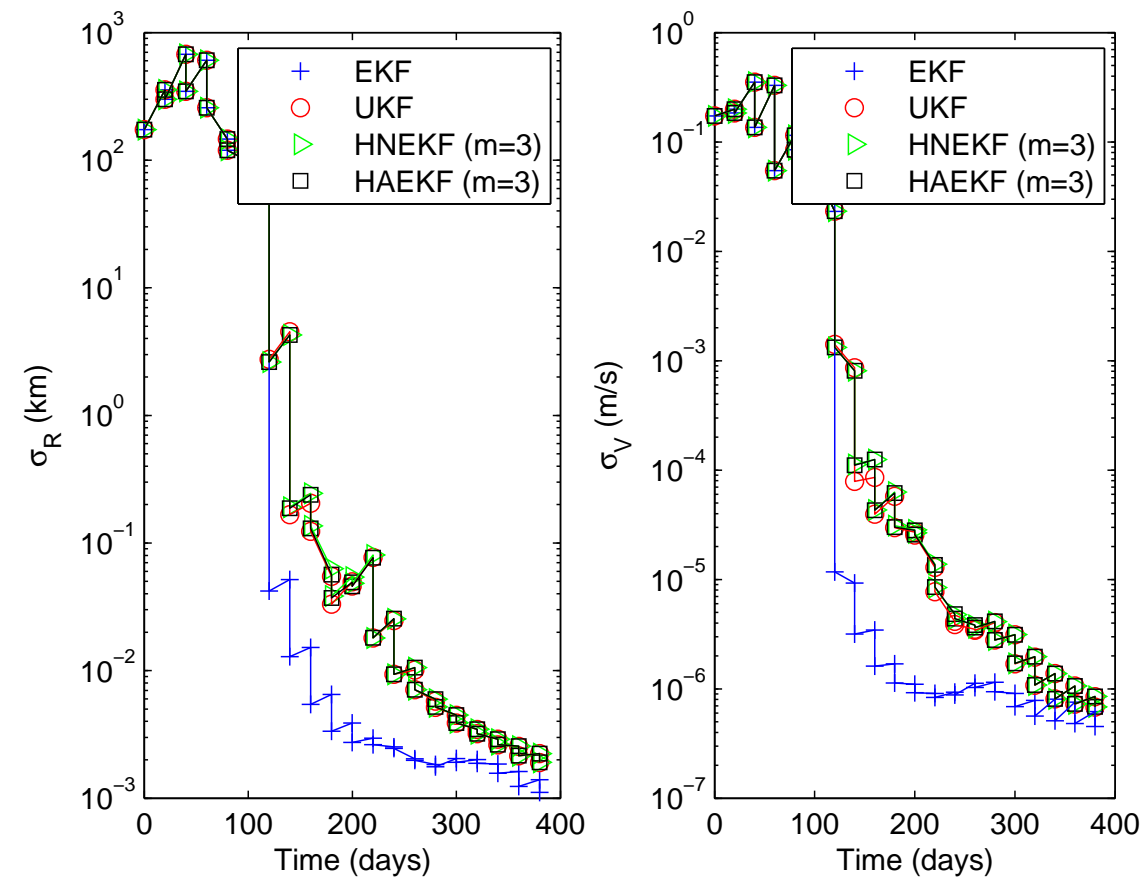

Figure 3. Comparison of the uncertainties computed using the EKF, UKF, HNEKF $(m=3)$, and HAEKF $(m=3)$. Measurements are taken every 20 days.
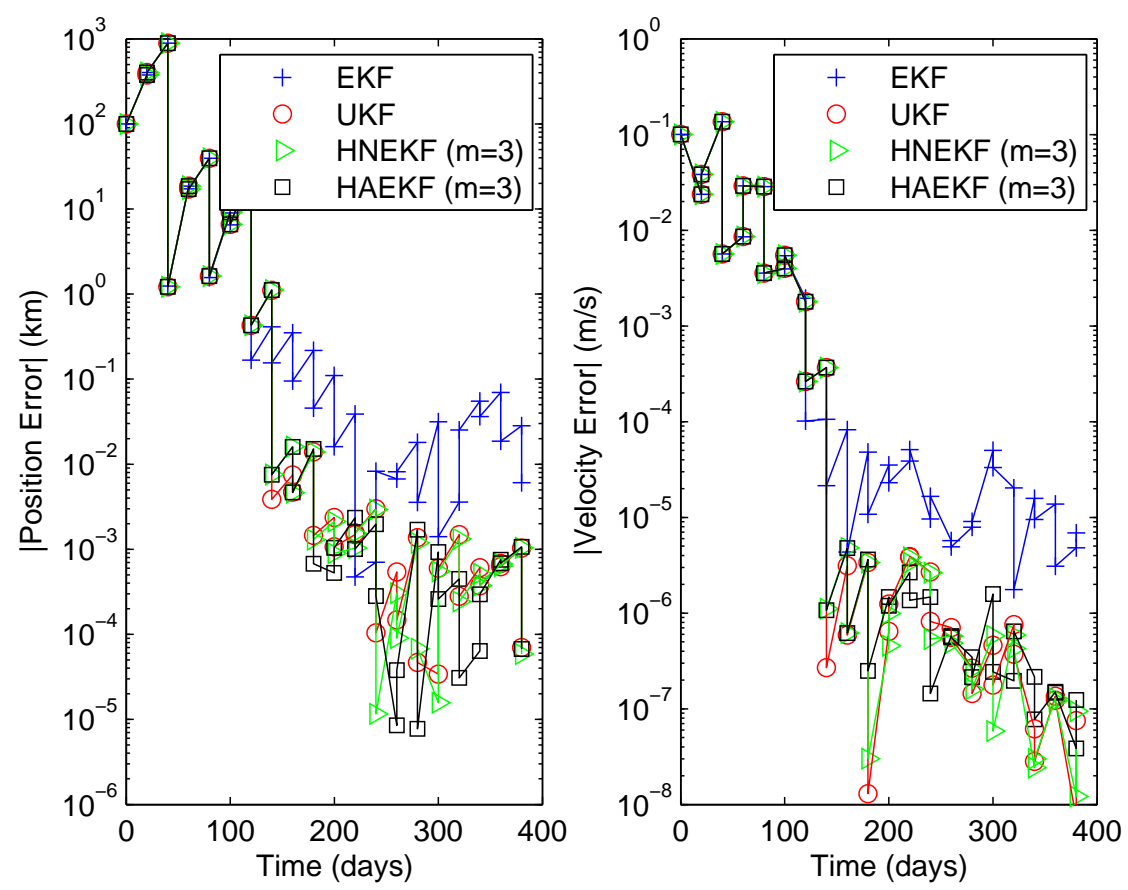

Figure 4. Comparison of the absolute error computed using the EKF, UKF, HNEKF $(m=3)$, and HAEKF $(m=3)$. Measurements are taken every 20 days. 

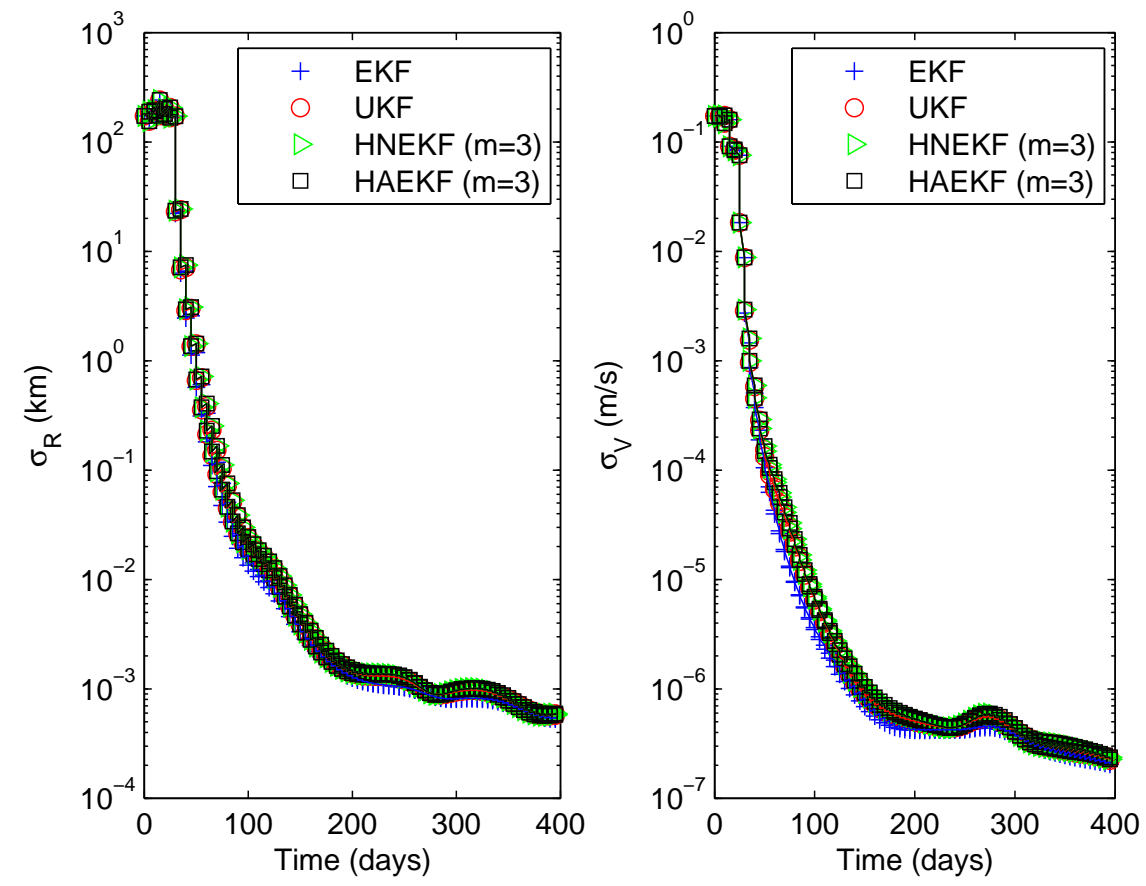

Figure 5. Comparison of the uncertainties computed using the EKF, UKF, HNEKF $(m=3)$, and HAEKF $(m=3)$. Measurements are taken every 5 days.
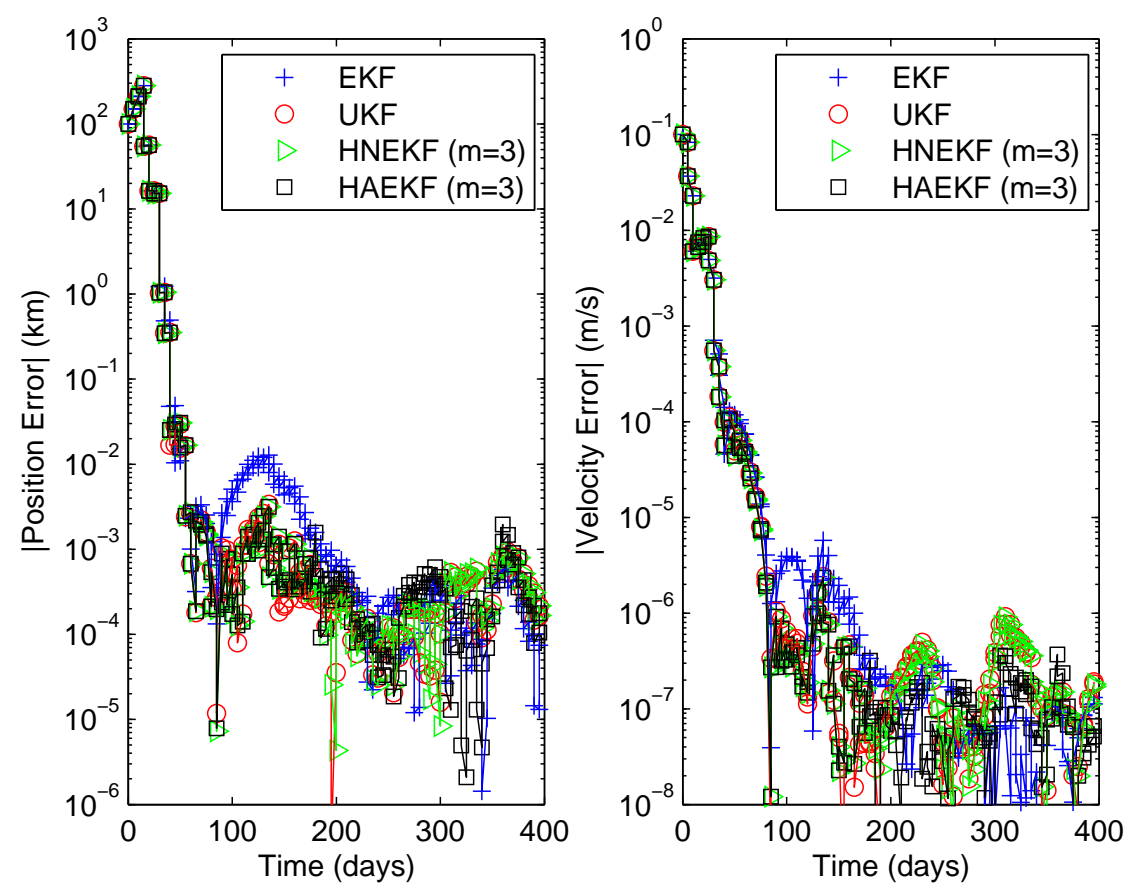

Figure 6. Comparison of the absolute error computed using the EKF, UKF, HNEKF $(m=3)$, and HAEKF $(m=3)$. Measurements are taken every 5 days. 

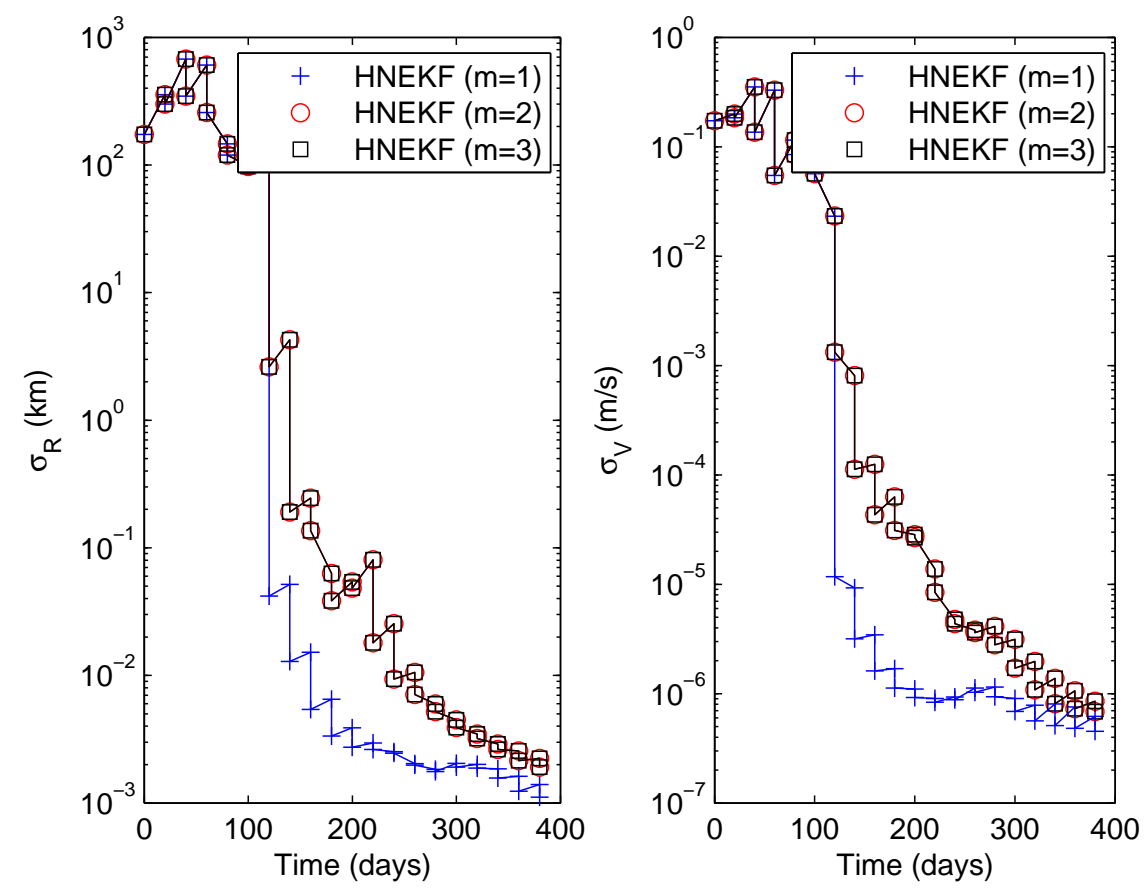

Figure 7. Comparison of the uncertainties computed using the HNEKFs for the cases $m=\{1,2,3\}$. Measurements are taken every 20 days.
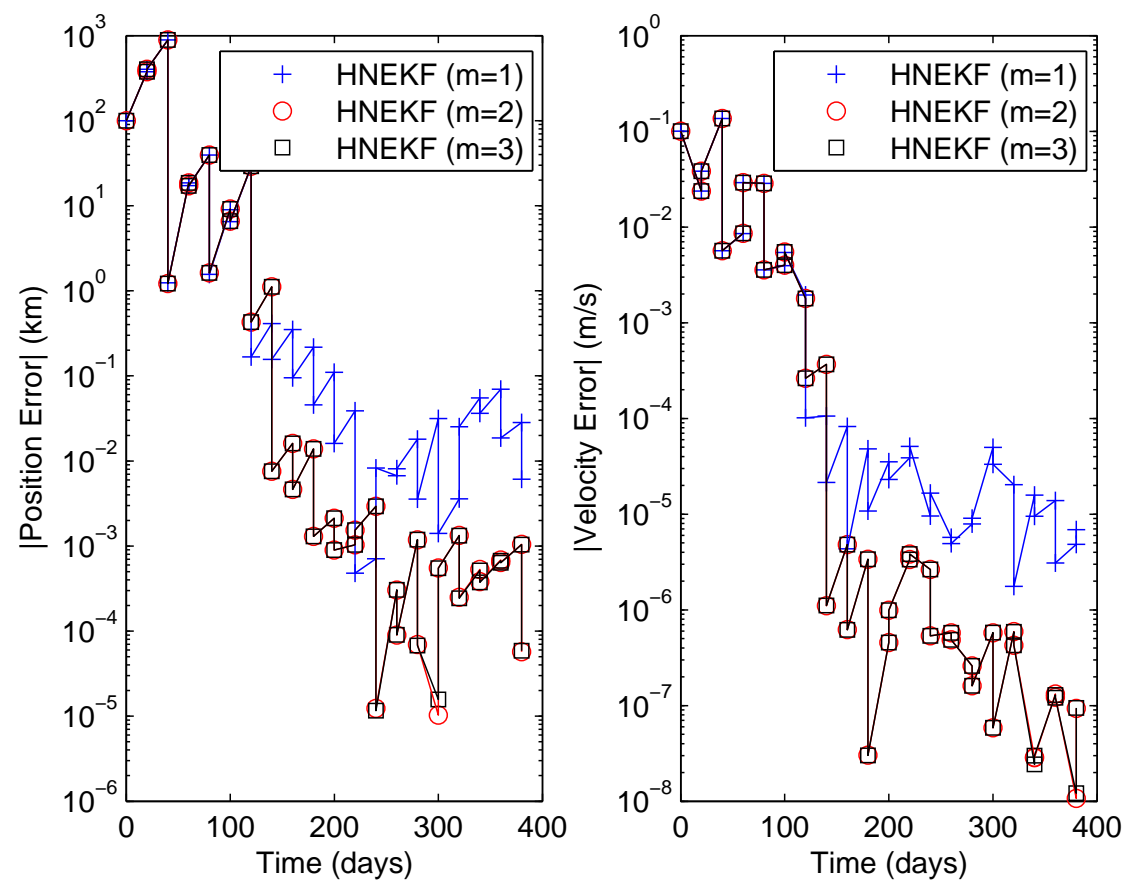

Figure 8. Comparison of the absolute errors computed using the HNEKFs for the cases $m=\{1,2,3\}$. Measurements are taken every 20 days. 

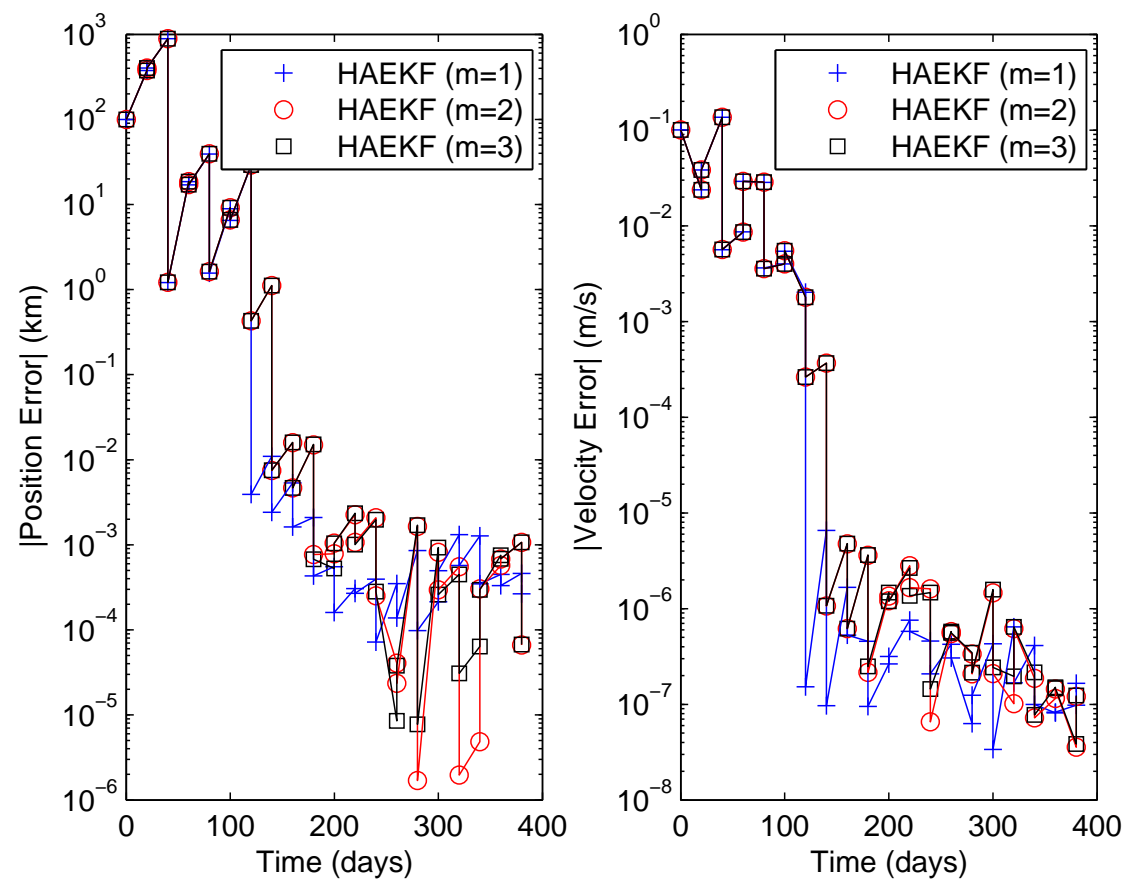

Figure 9. Comparison of the absolute errors computed using the HAEKFs for the cases $m=\{1,2,3\}$. Measurements are taken every 20 days.
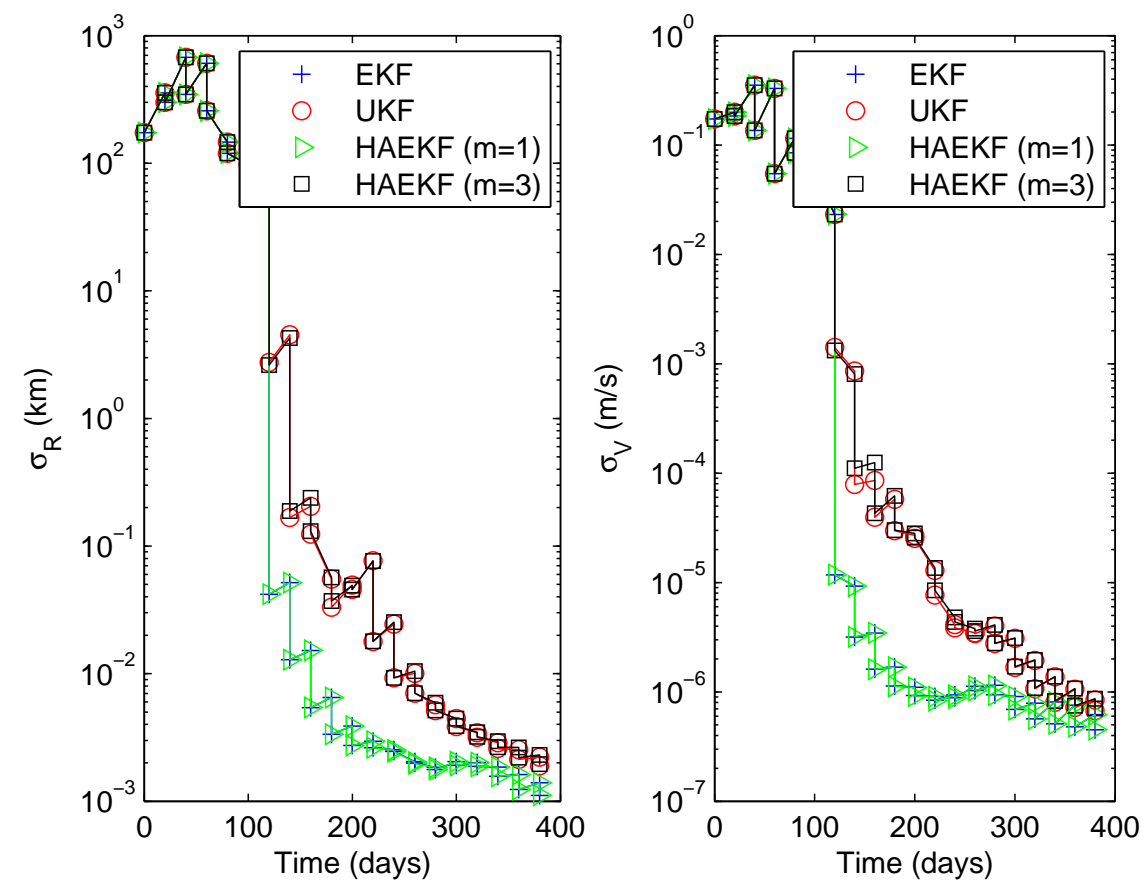

Figure 10. Comparison of the uncertainties computed using the EKF and HAEKFs for the cases $m=\{1,2,3\}$. Measurements are taken every 20 days based on the halo orbit case 2 . 



Figure 11. Comparison of the absolute errors computed using the EKF and HAEKFs for the cases $m=\{1,2,3\}$. Measurements are taken every 20 days based on the halo orbit case 2 . 